\title{
HUBBLE SPACE TELESCOPE AND GROUND-BASED OPTICAL AND ULTRAVIOLET OBSERVATIONS OF GRB 010222
}

\author{
T. J. Galama, ${ }^{1}$ D. Reichart,${ }^{1}$ T. M. Brown, ${ }^{2}$ R. A. Kimble,${ }^{3}$ P. A. Price, ${ }^{1}$ E. Berger, ${ }^{1}$ D. A. Frail, ${ }^{4}$ S. R. Kulkarni,${ }^{1}$ \\ S. A. Yost ${ }^{1}$ A. Gal-Yam, ${ }^{5}$ J. S. Bloom,${ }^{1}$ F. A. Harrison, ${ }^{1}$ R. SAri, ${ }^{6}$ D. Fox, ${ }^{1}$ and S. G. Djorgovski ${ }^{1}$ \\ Received 2002 June 25; accepted 2002 December 18
}

\begin{abstract}
We report on Hubble Space Telescope (HST) Wide Field Planetary Camera 2 optical and Space Telescope Imaging Spectrograph (STIS) near-ultraviolet MAMA observations and ground-based optical observations of GRB 010222, spanning $15 \mathrm{hr}$ to 71 days. The observations are well described by a relativistic blast wave model with a hard electron energy distribution, $p=1.57_{-0.03}^{+0.04}$, and a jet transition at $t_{*}=0.93_{-0.06}^{+0.15}$ days. These values are slightly larger than previously found as a result of a correction for the contribution from the host galaxy to the late-time ground-based observations and the larger temporal baseline provided by the $H S T$ observations. The host galaxy is found to contain a very compact core (size $<0$ ". 25$)$, which coincides with the position of the optical transient. The STIS near-ultraviolet MAMA observations allow for an investigation of the extinction properties along the line of sight to GRB 010222. We find that the far-ultraviolet curvature component $c_{4}$ is rather large. In combination with the low optical extinction, $A_{V}=0.110_{-0.021}^{+0.010}$ mag, when compared with the hydrogen column inferred from X-ray observations, we suggest that this is evidence for dust destruction.
\end{abstract}

Subject heading: gamma rays: bursts

\section{INTRODUCTION}

\section{1. $G R B 010222$}

On 2001 February 22 at 7:23:30 UT, the bright GRB 010222 was observed by the BeppoSAX Wide-Field Camera 1 (WFC; Piro 2001a). Within the 2!.5 error radius, an optical transient counterpart was identified at $\alpha=14^{\mathrm{h}} 52^{\mathrm{m}} 12.55$, $\delta=+43^{\circ} 01^{\prime} 06^{\prime \prime}$. (J2000.0; with an uncertainty of 0.2 ) (Henden 2001a; Henden \& Vrba 2001; McDowell et al. 2001). Soon this was followed by detections at X-ray (Piro 2001b), millimeter (Fich et al. 2001), and radio (Berger \& Frail 2001) wavelengths.

Absorption lines yielded a redshift of $z=1.4768 \pm 0.0002$ (Garnavich et al. 2001; Jha et al. 2001; Bloom et al. 2001; Castro et al. 2001; Masetti et al. 2001) for the highest redshift absorber. This system shows kinematic substructure, with two distinct systems at $z_{1}=1.47667 \pm 0.00005$ and $z_{2}=1.47755 \pm 0.00005$, corresponding to a rest-frame velocity separation of $106 \mathrm{~km} \mathrm{~s}^{-1}$, typical for internal motions in galaxies (Castro et al. 2001). The equivalent widths of the lines are unusually strong in comparison with metallic line absorbers seen in the spectra of quasars, indicating a high column density of gas, either suggesting a star-forming region environment or that the gamma-ray burst (GRB) occurred in the disk of the galaxy (or both).

\footnotetext{
${ }^{1}$ Division of Physics, Mathematics, and Astronomy, California Institute of Technology, MS 105-24, Pasadena, CA 91125.

2 Space Telescope Science Institute, 3700 San Martin Drive, Baltimore, MD 21218.

${ }^{3}$ Laboratory for Astronomy and Solar Physics, NASA Goddard Space Flight Center, Code 681, Greenbelt, MD 20771.

${ }^{4}$ National Radio Astronomy Observatory, P.O. Box O, Socorro, NM 87801.

${ }^{5}$ School of Physics and Astronomy, Tel Aviv University, Tel Aviv 69978 , Israel.

${ }^{6}$ California Institute of Technology, Theoretical Astrophysics 103-33, Pasadena, CA 91125.
}

The highest redshift absorber is therefore likely the host galaxy of the GRB itself (Jha et al. 2001). In addition, two foreground absorbing systems are detected (Bloom et al. 2001; Castro et al. 2001). These systems are typical for the metallic line absorbers at comparable redshifts. Further analysis of the two foreground absorbing systems is reported in Mirabal et al. (2002) and Salamanca et al. (2002). Frail et al. (2002) detected excess submillimeter emission toward GRB 010222, which they interpret as originating from a starburst host galaxy with $\mathrm{SFR} \sim 500 M_{\odot} \mathrm{yr}^{-1}$, much of which is obscured at optical wavelengths.

Optical observations have been presented by Cowsik et al. (2001), Lee et al. (2001), Masetti et al. (2001), Sagar et al. (2001), and Stanek et al. (2001). Although there is good agreement among these authors that the optical light curves exhibit an achromatic break about 0.5 days after the burst, no consensus has been reached regarding the origin of this steepening. Several authors have interpreted the optical afterglow in terms of the standard relativistic blast wave model (Sari, Piran, \& Narayan 1998). In this case, the break results from a collimated (jetted) outflow (Sari, Piran, \& Halpern 1999; Rhoads 1999); the synchrotron cooling frequency is below the optical passband, and the index $p$ of the electron energy distribution is unusually hard, $p \sim 1.4$ (Cowsik et al. 2001; Sagar et al. 2001; Stanek et al. 2001; Panaitescu \& Kumar 2002). Other authors prefer to interpret the break as a dynamical transition to nonrelativistic expansion (in 't Zand et al. 2001; Masetti et al. 2001), requiring a very energetic burst $\left(\sim 10^{54}\right.$ ergs $)$ expanding into a dense $\left(\sim 10^{6} \mathrm{~cm}^{-3}\right)$ circumburst medium. More recently, Björnsson et al. (2002) have argued in favor of the jet model but invoke continuous energy injection to explain why the standard model may erroneously be deriving a hard electron energy index.

Here we report on Hubble Space Telescope Wide-Field Planetary Camera 2 (WFPC2) and Space Telescope Imaging Spectrograph (STIS) near-ultraviolet (NUV) MAMA 
observations and ground-based optical observations. We interpret the resulting light curves in the context of a relativistic blast wave model with a hard electron energy distribution. The relatively large spectral baseline provided by the STIS NUV MAMA observations allows an investigation of the extinction properties along the line of sight to GRB 010222. In $\S 2$ we present the observations and the data analysis. In $\S 3$ we present the optical light curves, model fits to the data, and investigate the extinction properties along the line of sight. In $\S 4$ we discuss the results and conclude.

\section{OBSERVATIONS AND DATA REDUCTION}

\subsection{HST STIS Near-Ultraviolet MAMA Observations}

On 2001 February 26.15 UT, the optical transient (OT) was imaged with the STIS using the NUV MAMA and the F25QTZ filter. This filter spans 1450-3500 A (thus excluding terrestrial airglow lines of $\mathrm{Ly} \alpha$ and $\mathrm{O}$ I), but little source flux is expected below the redshifted Lyman limit of the OT $(2253 \AA$ ). We obtained six exposures spanning $2.42-4.64 \mathrm{hr}$ UT, with exposure times of 800-866 s. The OT was placed near the center of the detector in each exposure, but dithering of 5-16 pixels placed the OT at a different location in each frame to smooth over small-scale variations in sensitivity.

The raw STIS exposures were reduced using the CALSTIS package of the STIS Instrument Definition Team (IDL; Lindler 1999). ${ }^{7}$ Using IDL, we performed aperture photometry on each frame assuming a source aperture of 10 pixel radius $(0$ ".25) and a sky annulus spanning radii 30-50 pixels. The OT is well detected in each frame, at a signal-to-noise ratio greater than 10 . Summing the net counts in each frame and dividing by the total exposure time yields a mean count rate of $0.323 \pm 0.012$ counts $\mathrm{s}^{-1}$. There is no evidence for variability over the $2 \mathrm{hr}$ of STIS exposures; the count rate measured in each frame scatters within $1 \sigma$ around the mean rate. The encircled energy within the source aperture is 0.856 for NUV MAMA imaging at these wavelengths (Robinson 1997) $;{ }^{8}$ with an aperture correction, the measured count rate in the STIS bandpass is thus $0.377 \pm 0.014$ counts $\mathrm{s}^{-1}$. To convert the STIS count rate to flux at a given wavelength requires the assumption of a spectral energy distribution (SED), a discussion of which we present in $\S 3.2$.

We co-added the individual exposures using the DRIZZLE package (Fruchter \& Hook 2002) to determine whether other faint objects are visible in the STIS image. The co-addition was done with a mask for hot and bad pixels and included a correction for the geometric distortion in the STIS camera. Three extended objects lie near the OT; these objects are also well detected in the WFPC2 images (see Fig. 1). We performed aperture photometry on these objects assuming a source aperture radius of 50 pixels and a sky annulus spanning 50-70 pixels. The objects are noncircular in shape, but this source aperture encloses the detectable flux from each. The first source lies 7"!7 south of the OT and has a count rate of $0.83 \pm 0.06$ counts $^{-1}$; the second source lies 7". 6 southeast of the OT, with a count rate of $0.30 \pm 0.06$ counts $\mathrm{s}^{-1}$; the third source lies 4.22 north-

\footnotetext{
${ }^{7}$ Additional information can be found at http://hires.gsfc.nasa.gov/ stis/docs/calstis/.

${ }^{8}$ Additional information can be found at http://hires.gsfc.nasa.gov/ stis/postcal/quick_reports.
}

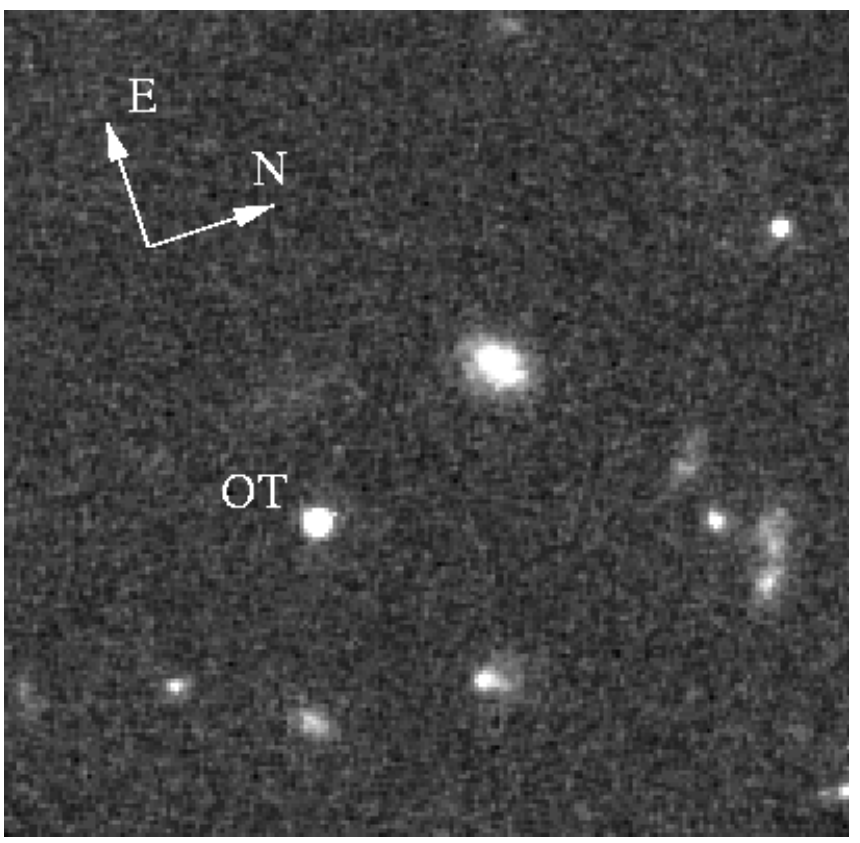

FIG. 1.- Shows F606W drizzled image (each pixel is 0. "071) of a region around the optical transient (OT). The OT and the galaxy to the northeast are separated by 3 ".96.

northeast of the OT, with a count rate of $0.20 \pm 0.06$ counts $\mathrm{s}^{-1}$. Without any knowledge about the SEDs, these count rates cannot be converted to fluxes accurately, but it is worth noting that in the STIS bandpass, these objects are of a brightness comparable to that of the OT.

\subsection{HST WFPC2 Observations}

We obtained WFPC2 HST observations in the F450W, F606W, and F814W passbands at four epochs as part of an HST cycle 9 program. These observations took place between 2001 February 28.66 UT and May 4.73 UT (6.3571.42 days after the GRB). The transient was positioned at the WFALL position on WFPC2 CCD 3. A log of the observations is provided in Table 1 .

TABLE 1

WFPC2 Hubble Space Telescope OBSERVATIONS OF GRB 010222

\begin{tabular}{|c|c|c|c|}
\hline $\begin{array}{c}\text { Date } \\
\text { (2001 UT) }\end{array}$ & Filter & $\begin{array}{l}\text { Exposure Time } \\
\text { (s) }\end{array}$ & Magnitude \\
\hline Feb $28.66 \ldots \ldots$. & F450W & $2 \times 1100(1$ orbit $)$ & $23.463 \pm 0.034$ \\
\hline Feb $28.75 \ldots \ldots$. & F606W & $4 \times 1100(2$ orbits $)$ & $23.029 \pm 0.010$ \\
\hline Feb $28.89 \ldots \ldots .$. & F814W & $4 \times 1100(2$ orbits $)$ & $22.440 \pm 0.012$ \\
\hline Mar $17.86 \ldots \ldots$. & F450W & $2 \times 1100(1$ orbit $)$ & $25.48 \pm 0.15$ \\
\hline Mar $17.96 \ldots \ldots$. & F606W & $4 \times 1100(2$ orbits $)$ & $25.075 \pm 0.029$ \\
\hline Mar $18.90 \ldots . .$. & F814W & $4 \times 1100(2$ orbits $)$ & $24.41 \pm 0.05$ \\
\hline Apr $5.40 \ldots \ldots \ldots$ & F450W & $2 \times 1100(1$ orbit $)$ & $25.71 \pm 0.16$ \\
\hline Apr $5.49 \ldots \ldots \ldots$ & F606W & $4 \times 1100(2$ orbits $)$ & $25.72 \pm 0.04$ \\
\hline Apr $6.56 \ldots \ldots \ldots$ & F814W & $4 \times 1100(2$ orbits $)$ & $25.02 \pm 0.06$ \\
\hline May $4.35 \ldots \ldots . .$. & F606W & $6 \times 1000(3$ orbits $)$ & $25.96 \pm 0.06$ \\
\hline May $4.73 \ldots \ldots . .$. & F814W & $4 \times 1100(2$ orbits $)$ & $25.37 \pm 0.08$ \\
\hline Sep $8.38 \ldots \ldots \ldots . .$. & F450W & $6 \times 1000(3$ orbits $)$ & $25.94 \pm 0.10$ \\
\hline Sep $8.59 \ldots \ldots \ldots . .$. & F606W & $6 \times 1000(3$ orbits $)$ & $25.82 \pm 0.09$ \\
\hline Sep $9.39 \ldots \ldots \ldots . .$. & F814W & $6 \times 1000(3$ orbits $)$ & $25.53 \pm 0.15$ \\
\hline
\end{tabular}

NoTE.--Reported magnitudes are for a 5 pixel aperture. 
TABLE 2

Transformed WFPC2 Hubble Space Telescope Magnitudes to Johnson-Cousins System

\begin{tabular}{ccccc}
\hline \hline $\begin{array}{c}\text { Date } \\
(2001 \mathrm{UT})\end{array}$ & $B$ & $V$ & $R$ & $I$ \\
\hline Feb $28.75 \ldots \ldots \ldots \ldots . .$. & $23.544 \pm 0.038$ & $23.264 \pm 0.017$ & $22.818 \pm 0.015$ & $22.418 \pm 0.015$ \\
Mar $17.96 \ldots \ldots \ldots \ldots$. & $25.55 \pm 0.16$ & $25.35 \pm 0.04$ & $24.84 \pm 0.04$ & $24.39 \pm 0.05$ \\
Apr $5.49 \ldots \ldots \ldots \ldots \ldots$. & $25.69 \pm 0.16$ & $26.01 \pm 0.05$ & $25.47 \pm 0.06$ & $25.01 \pm 0.06$ \\
May $4.54 \ldots \ldots \ldots \ldots . .$. & $\ldots$ & $26.20 \pm 0.07$ & $25.75 \pm 0.08$ & $25.35 \pm 0.08$ \\
Sep $8.79 \ldots \ldots \ldots \ldots \ldots$. & $25.96 \pm 0.10$ & $25.92 \pm 0.10$ & $25.72 \pm 0.13$ & $25.52 \pm 0.15$ \\
\hline
\end{tabular}

The F450W images were combined using the STSDAS task CRREJ. The F606W and F814W images at each epoch were observed at two offsets, offset by $+2.5,+2.5$ pixels in $x$ and $y$. These images were combined and cosmic-rayrejected using the DRIZZLE technique (Fruchter \& Hook 2002). The drizzled images have pixels half the area of the original WFPC2 data. We determined the magnitude of the optical transient (OT) in a 2 pixel aperture radius $(2.83$ pixels for the drizzled images) and determined the aperture correction from 2 to 5 pixels (7.1 pixels for the drizzled images) to obtain the corresponding 5 pixel radius magnitude. We corrected the magnitudes for geometric distortion of the images (Holtzman et al. 1995; a correction of 0.024 mag) and for nonoptimal charge transfer efficiency (CTE; less than $0.023 \mathrm{mag}$ ). Next, we calibrated the WFPC2 data using the zero points and color transformations to the Johnson-Cousins system in Table 10 of Holtzman et al. (1995). These magnitudes are given in Tables 1 and 2. The quoted errors are statistical only; we estimate the uncertainty in the absolute calibration to be about 0.05 in $B, V, R$, and $I$.

The F606W drizzled image of a region around the OT of February 28.75 UT can be seen in Figure 1. The three objects visible in the STIS NUV-MAMA images are also identified in the WFPC2 images, although one of the objects falls on the edge of the CCD. The galaxy 3".96 northeast of the OT is the only one of those three that is displayed. In addition, several other galaxies are seen.

We obtain curves of growth to measure the magnitudes of the host in the WFPC2 images; correcting for the contribution of the OT and the contribution of the galaxy in a 2 pixel radius (see $\S 3.2$ ), we find $B_{\text {gal }}=25.71_{-0.24}^{+0.32}, V_{\text {gal }}=$ $26.18_{-0.13}^{+0.15}, R_{\mathrm{gal}}=26.42_{-0.21}^{+0.27}$, and $I_{\mathrm{gal}}=25.66_{-0.24}^{+0 . \overline{3}^{0}} \mathrm{mag}$.

In the final epoch, $H S T$ images the flux measured at the OT position is dominated by the host galaxy emission. Figure 2 shows the WFPC2 F606W fluxes extracted with increasing apertures, and normalized to unity at 1 !" 0 , for the host emission (diamonds) compared with a reference star (triangles). The figure shows evidence for a very compact emission component, $30 \%-50 \%$ of which can be attributed to the optical transient, and an additional low-level extended emission component.

A cross correlation of epoch 1 and epoch 6 F606W imaging data allows for us to place the OT position, measured using IRAF/CENTER/OFILTER in epoch 1, atop the apparent host galaxy in epoch 6 (see Bloom, Kulkarni, \& Djorgovski 2002a for details). The host center was determined using a centroid algorithm, assuming that the light in epoch 6 is dominated by the host. We determine the offset of the optical transient from the host galaxy to be $42.9 \pm 5.6$ mas east and $7.8 \pm 5.8$ mas south, i.e., a total offset of
$43.6 \pm 5.6$ mas. This amounts to an offset of $390 \pm 51 \mathrm{pc}$ in projection at the redshift of GRB 010222 and places the offset of GRB 010222 in the $20 \%$ percentile of observed GRB offsets to date (Bloom et al. 2002a). Note that, since the offset is smaller than the resolution of final images, if the OT contributes some of the light to the core of the host in epoch 6 , probably not more than $30 \%$, then the true offset could be larger by not more than a factor of $\sim 1.3$.

\subsection{Ground-based Optical Observations}

Following the identification of the optical afterglow, we commenced multicolor observations with the Wise Observatory $1 \mathrm{~m}$ telescope. The Wise observations were made with the SITe $2 \mathrm{k} \times 4 \mathrm{k}$ CCD using on-chip $2 \times 2$ binning, resulting in a pixel scale of 0 ". 8 pixel $^{-1}$. The observations lasted until about 3 days after the burst. Observations from the Palomar Observatory were hampered by poor weather, but we obtained observations $4.2 \mathrm{hr}$ after the event in a Sloan $r^{\prime}$ filter with the 200 inch Hale telescope using the large format camera (LFC) and in $B, V, R$, and $I$ on 2001 March 2.468 UT using the Jacobs camera (JCAM). The LFC consists of six $2 \mathrm{k} \times 4 \mathrm{k}$ SITe, thinned, backside-illuminated CCDs, placed in a symmetric cross-shaped pattern, providing a roughly $24^{\prime}$ diameter field of view (FOV).

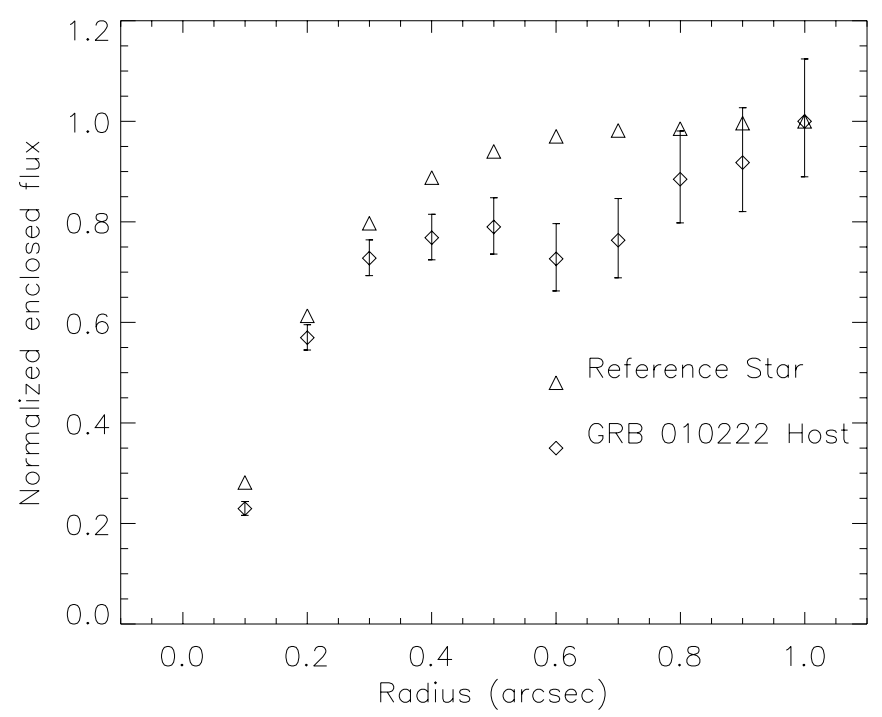

FIG. 2.-Emission from a region around the optical transient (OT) in the September $8 H S T$ F606W drizzled image as a function of extraction radius (diamonds) compared with a reference star (triangles). The vertical scale is the enclosed flux normalized to unity at a 1 ".0 radius. The figure shows evidence for a compact knot of emission (presumably the core of the host galaxy) and evidence for a low-level extended emission component, when compared with the instrument point-spread function. 
TABLE 3

Ground-Based Optical ObSERVATIONS OF GRB 010222

\begin{tabular}{|c|c|c|c|}
\hline $\begin{array}{c}\text { Date } \\
\text { (2001 UT) }\end{array}$ & Passband & Magnitude & Telescope \\
\hline Feb 22.948 . & $R$ & $19.387 \pm 0.098$ & Wise $1 \mathrm{~m}$ \\
\hline Feb 22.959 . & $R$ & $19.567 \pm 0.082$ & Wise $1 \mathrm{~m}$ \\
\hline Feb $22.970 \ldots \ldots \ldots \ldots$ & $V$ & $20.052 \pm 0.085$ & Wise $1 \mathrm{~m}$ \\
\hline Feb $23.063 \ldots \ldots \ldots \ldots$ & $I$ & $19.102 \pm 0.047$ & Wise $1 \mathrm{~m}$ \\
\hline Feb $23.072 \ldots$ & $R$ & $19.676 \pm 0.053$ & Wise $1 \mathrm{~m}$ \\
\hline Feb $23.086 \ldots \ldots \ldots \ldots$ & $V$ & $20.093 \pm 0.061$ & Wise $1 \mathrm{~m}$ \\
\hline Feb $24.020 \ldots \ldots \ldots \ldots$ & $R$ & $20.859 \pm 0.075$ & Wise $1 \mathrm{~m}$ \\
\hline Feb $24.052 \ldots \ldots \ldots \ldots$ & $V$ & $21.279 \pm 0.109$ & Wise $1 \mathrm{~m}$ \\
\hline Feb $24.084 \ldots \ldots \ldots \ldots$ & $I$ & $20.582 \pm 0.118$ & Wise $1 \mathrm{~m}$ \\
\hline Feb $25.085 \ldots \ldots \ldots \ldots$ & $R$ & $21.607 \pm 0.129$ & Wise $1 \mathrm{~m}$ \\
\hline Feb $25.136 \ldots \ldots \ldots \ldots$ & $V$ & $21.721 \pm 0.231$ & Wise $1 \mathrm{~m}$ \\
\hline Feb $22.482 \ldots \ldots \ldots \ldots$ & $R$ & $18.455 \pm 0.033$ & P200 LFC \\
\hline Feb $22.487 \ldots \ldots \ldots \ldots$ & $R$ & $18.481 \pm 0.036$ & P200 LFC \\
\hline Mar 2.468 ............. & $I$ & $22.57 \pm 0.35$ & P200 JCAM \\
\hline Mar 2.468 .............. & $R$ & $23.19 \pm 0.15$ & P200 JCAM \\
\hline Mar $2.468 \ldots \ldots \ldots \ldots . .$. & $V$ & $23.54 \pm 0.17$ & P200 JCAM \\
\hline Mar 2.468 .............. & $B$ & $24.15 \pm 0.46$ & P200 JCAM \\
\hline
\end{tabular}

Note.-P200 LFC images were obtained with Steidel $R$ filter.

JCAM is a dual CCD imager with a 3.2 diameter FOV (Bloom et al. 2003). The observations were reduced in the standard manner and photometered relative to several field stars calibrated by Henden (2001b). The errors in the table reflect the statistical and systematic uncertainties, the latter of which were sizable for the JCAM and LFC observations because of the lack of suitable secondary standards that did not allow us to fit reliable color terms. We therefore photometered the afterglow relative to field stars with colors similar to the OT, without applying any color correction. A log of the observations is presented in Table 3 .

\section{AFTERGLOW MODEL}

\subsection{The Optical Light Curves}

We supplement the HST WFPC2 and ground-based optical observations from Tables 2 and 3 with optical and infrared observations from Cowsik et al. (2001), Lee et al. (2001), Masetti et al. (2001), Sagar et al. (2001), Stanek et al. (2001), and Watanabe et al. (2001), and with those reported in several GRB Coordinate Network (GCN) circulars (Orosz 2001; Massi, Dolci, \& Carlo 2001; Oksanen et al. 2001; Veillet 2001a, 2001b; Holland et al. 2001). We restricted the data set to observations for which magnitudes were reported relative to field stars and converted those to the calibration of Henden (2001b) and corrected for a small foreground Galactic extinction $\left(E_{B-V}=0.023 \mathrm{mag}\right.$ from Schlegel, Finkbeiner, \& Davis 1998). The infrared measurements of the OT by Masetti et al. (2001) were taken in poor seeing $\left(3^{\prime \prime}-4^{\prime \prime}\right)$, and the authors therefore used an aperture of $5^{\prime \prime}$ in radius. Keck $K$-band observations presented in Frail et al. (2002) show that there are several other (relatively bright) near-infrared (NIR) sources nearby. Within this aperture we measure $K=17.57 \pm 0.11$, very comparable to what is measured by Masetti et al. (2001). We conclude that the NIR measurements of Masetti et al. (2001) are severely contaminated (thus explaining the flat NIR light curve), and we do not include these measurements in our data set. Because the late-time ground-based optical data are also

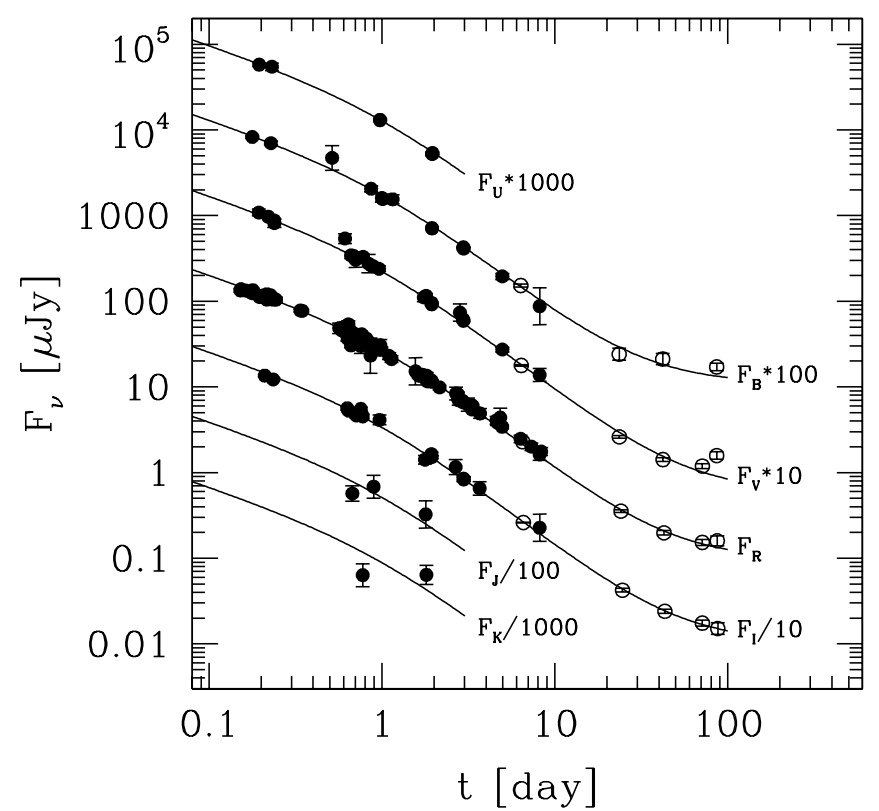

FIG. 3. - Shows the UBVRIJK band light curves of GRB 010222. The $H S T$ data are shown by open symbols, while the ground-based data are shown with filled symbols. Shown are fits of eq. (1) to the data: a smoothly broken temporal power law with a break time of $t_{*}=0.93_{-0.06}^{+0.15}$ days and late-time temporal index $\alpha_{2}=p=1.57$, assuming a power-law spectrum with index $\beta=p / 2$ and the full extinction curve of Reichart (2001). The late-time flattening is due to a host contributing to the flux in the HST WFPC2 images in a 2 pixel aperture and is modeled by a constant contribution. For details, see $\S 3.2$.

significantly contaminated by the host galaxy, we have not included two $R$-band data points 10 days after GRB 010222. We converted the Sloan Digital Sky Survey $u^{\prime}, g^{\prime}, i^{\prime}$, $r^{\prime}$, and $z^{\prime}$ observations (Lee et al. 2001) to $U, B, V, R$, and $I$ using the transformations of Fukugita et al. (1996), assuming no fading over the course of the $2.5 \mathrm{~m}$ observations (predicted fading is $0.005 \mathrm{mag}$, which is negligible) and a constant color for the $1.5 \mathrm{~m}$ observations of $\left(g^{\prime}-r^{\prime}\right)=0.28 \pm 0.03 \mathrm{mag}$. An additional 3\% transformation error was added in quadrature to the statistical error for these measurements.

In Figure 3 we display the optical light curves. We have added an additional $0.042 \mathrm{mag}$ error in quadrature, the socalled slop parameter $\sigma$ (see $\S 3.2$ ), to reflect uncertainties in the calibration between different instruments. The magnitudes have been converted to flux densities using the transformations of Bessell (1979) and Bessell \& Brett (1988). The late-time HST (WFPC2) observations show that the optical light curves, after a shallow break around 0.7 days, continue roughly at the same decay rate $F_{\nu} \propto t^{-1.4}$, as previously found by ground-based observations (Cowsik et al. 2001; Masetti et al. 2001; Stanek et al. 2001; Sagar et al. 2001), and show a leveling off to a constant value in the $B, V, R$, and $I$ band around 25 days (we cannot establish the presence of a host component in the $U$ and infrared passbands due to a lack of late-time observations in those bands). The former has been interpreted as the result of a jet-break transition with a very hard electron energy distribution $p \sim 1.4$ (Masetti et al. 2001; Sagar et al. 2001). The latter is simply interpreted as due to emission from the host galaxy of GRB 010222 in the 2 pixel radius aperture, in which we determined the mag of the OT (WFPC2). This is consistent with 
the fact that the source is extended in the WFPC2 images in the later epochs. Therefore, we also have to correct the ground-based data for the contribution of the host outside the 2 pixel radius aperture we used for the WFPC2 images. We determine the difference between a 2 pixel and a large aperture from the HST WFPC2 observations and corrected the ground-based data accordingly.

\subsection{Fits to the Broadband GRB 010222 Light Curves}

We now model the optical/NIR photometry and STIS count rate and constrain model parameters. We model the spectrum with a power law that is extinguished by dust in the host galaxy and our Galaxy and absorbed by hydrogen in the host galaxy and Ly $\alpha$ forest, and we model the light curve with a smooth achromatic, broken power law added to a constant, presumably host galaxy, component,

$$
\begin{aligned}
F_{\nu}(t)= & e^{-\tau_{\nu}^{\mathrm{MW}}} e^{-\tau_{\nu(1+z)}^{\mathrm{Ly} \alpha}}\left\{e^{-\tau_{\nu(1+z)}^{\mathrm{host}}} F_{0}\left(\frac{\nu}{\nu_{R}}\right)^{\beta}\right. \\
& \left.\times\left[\left(\frac{t}{t_{*}}\right)^{-\alpha_{1} s}+\left(\frac{t}{t_{*}}\right)^{-\alpha_{2} s}\right]^{-1 / s}+F_{\nu}^{\text {host }}\right\},
\end{aligned}
$$

where $\tau_{\nu}^{\mathrm{MW}}$ is the Galactic extinction curve model of Cardelli, Clayton, \& Mathis (1989), $\tau_{\nu(1+z)}^{\mathrm{Ly} \alpha}$ is the Ly $\alpha$ forest absorption model of Reichart $(2001), \tau_{\nu(1+z)}^{\text {host }}$ is the host galaxy extinction curve and Lyman limit absorption model of Reichart (2001), $F_{0}$ is a normalization parameter, $\nu_{R}$ is the effective frequency of the $R$ band, $\beta$ is the spectral slope, $t_{*}$ is the light-curve break time, $s$ is the break smoothness parameter, $\alpha_{1}$ is the limiting temporal slope before $t_{*}, \alpha_{2}$ is the limiting temporal slope after $t_{*}$, and $F_{\nu}^{\text {host }}$ is the spectral flux of the host galaxy at frequency $\nu$. The STIS bandpass samples 912-1400 $\AA$ in the rest frame, and so $\mathrm{H}$ and $\mathrm{H}_{2}$ absorption lines are expected to be present in the actual flux distribution; however, a smooth power law suffices to describe the flux in the broad STIS bandpass. We use the full extinction/absorption model of Reichart (2001), because Lee et al. (2001) find a nonstandard extinction curve in the source-frame far-ultraviolet (FUV; see below), and the STIS NUV MAMA transmission function spans the sourceframe FUV, Ly $\alpha$ forest, and Lyman limit.

Since the source-frame UV extinction curve model has features that are narrower than most photometric bands, we convolve equation (1) with a boxcar approximation to the appropriate filter function before fitting it to the optical/ NIR photometry. We have found that failure to do this can cause one to erroneously identify LMC- and SMC-like extinction curves when an extinction curve with a large FUV excess component, possibly related to the fragmentation of grains by the burst (see below), is actually favored by the data. In the case of the STIS measurement, we fitted

$$
C=A \int_{0}^{\infty} \frac{F_{\nu}\left(t_{\mathrm{STIS}}\right)}{h \nu} T(\nu) d \nu
$$

to the measured count rate, where $A$ is the collecting area of the mirror and $T(\nu)$ is the transmission function of the mirror and instrument.

Following previous efforts (Cowsik et al. 2001; Sagar et al. 2001; Stanek et al. 2001; Panaitescu \& Kumar 2002), we chose to fit the data in terms of the standard afterglow model (e.g., Sari et al. 1998, 1999). We do not favor an interpretation in which the break is due to a transition of the blast wave to the nonrelativistic phase (in 't Zand et al. 2001; Masetti et al. 2001). The same claim has been made for GRB 000926 (Piro et al. 2001), but the high ambient density causes the self-absorption frequency to lie far above the radio passband (Harrison et al. 2001), resulting in an undetectable radio afterglow, contrary to observations. The jet model with continuous energy injection (Björnsson et al. 2002) is an interesting alternative, but adequate testing of this model will require a full broadband data set.

A significant difference between our model fit and others is that $\alpha_{1}$ and $\alpha_{2}$ are not independent but are in fact directly related to each other by the index $p$ of the electron energy distribution. Previous fits to the GRB 010222 light curves have treated these two parameters as independent (e.g., Stanek et al. 2001). We take $\alpha_{1}=-(3 p-2) / 4, \alpha_{2}=-p$, and $\beta=-p / 2$ (where $p$ is the power-law index of the electron energy distribution), a relation that applies in the standard jet model when the optical band lies above the synchrotron cooling frequency (Sari et al. 1999). This relation may not be obeyed when $1 \leq p \leq 2$ (Bhattacharya 2001; Panaitescu 2001; Dai \& Cheng 2001). In order to avoid divergence of the shock energy, an additional cutoff $\gamma_{u}$ in the electron energy distribution must be introduced. In general, this will modify the temporal dependence of the decay indices, compared with the $p>2$ case. However, Bhattacharya (2001) has shown that the behavior is identical when $\gamma_{u}$ varies in direct proportion to the bulk Lorentz factor of the shock. Although this is a reasonable assumption, we note that other solutions are possible, depending on what is adopted for the evolution of $\gamma_{u}$.

We fitted the standard model to the optical/NIR photometry and STIS NUV MAMA count rate using Bayesian inference (e.g., Reichart 2001); the posterior probability distribution is equal to the product of the prior probability distribution and the likelihood function. The likelihood function is given by

$$
L=\prod_{i=1}^{N} \frac{1}{\left[2 \pi\left(\sigma_{i}^{2}+\sigma^{2}\right)\right]^{1 / 2}} \exp \left\{-\frac{1}{2} \frac{\left[y\left(\nu_{i}, t_{i}\right)-y_{i}\right]^{2}}{\sigma_{i}^{2}+\sigma^{2}}\right\},
$$

where $N$ is the number of measurements; $y\left(\nu_{i}, t_{i}\right)$ is the above described convolution of equation (1) evaluated at the effective frequency and time of the $i$ th measurement if $i<N$, where $y\left(\nu_{i}, t_{i}\right)=C$ (eq. [2]) if $i=N$; $y_{i}$ is the $i$ th measurement in units of $\log$ spectral flux if $i<N$ and $\log$ count rate if $i=N ; \sigma_{i}$ is the uncertainty in the $i$ th measurement in the same units; and $\sigma$ is a parameter, sometimes called the slop parameter, that models the small systematic errors that are unavoidably introduced when data are collected from a wide variety of sources, and other small sources of error (Reichart 2001).

Many of the parameters of the host galaxy extinction curve model, and all of the parameters of the Ly $\alpha$ forest absorption model and Galactic extinction curve model, can be constrained a priori. The host galaxy extinction curve model of Reichart (2001) is a function of eight parameters: the source-frame $V$-band extinction magnitude $A_{V}$, $R_{V}=A_{V} / E(B-V)$, the intercept $c_{1}$ and slope $c_{2}$ of the linear component of the source-frame UV extinction curve, the strength $c_{3}$, width $\gamma$, and center $x_{0}$ of the UV bump component of the extinction curve, and the strength $c_{4}$ of the FUV excess component of the extinction curve. The Ly $\alpha$ forest model of Reichart (2001) is a function of a single 
parameter, $D_{A}$, the flux deficit. Reichart (2001) finds prior probability distributions for $R_{V}, c_{1}, \gamma, x_{0}$, and $D_{A}$, which means that the values of these parameters can be weighted by fairly narrow distributions, the parameterization of which sometimes depends on other parameters (in particu$\operatorname{lar} c_{2}$ and $z$ ), a priori. We adopt these priors here, which can be thought of as increasing the degrees of freedom by 5 . Also, the Galactic extinction curve model of Cardelli et al. (1989) is a function of a single parameter, $R_{V}^{\mathrm{MW}}$. We adopt a prior for this parameter that is log normally distributed with mean $\log (3.1)$ and width 0.1 , which closely approximates the distribution of values of this parameter along random lines of sight through the Galaxy (e.g., Reichart 2001).

The best fit is found by maximizing the posterior. We find that $F_{0}=60.9_{-5.0}^{+10.2} \mu \mathrm{Jy}, p=1.57_{-0.03}^{+0.04}, t_{*}=0.93_{-0.06}^{+0.15}$ days, $s=1.51_{-0.08}^{+0.08}, \quad F_{B}^{\text {host }}=0.109_{-0.013}^{+0.014} \mu \mathrm{Jy}, \quad F_{V}^{\text {host }}=0.103_{-0.011}^{+0.011}$ $\mu \mathrm{Jy}, \quad F_{R}^{\text {host }}=0.063_{-0.008}^{+0.008} \quad \mu \mathrm{Jy}, \quad F_{I}^{\text {host }}=0.119_{-0.024}^{+0.027} \quad \mu \mathrm{Jy}$, $A_{V}=0.110_{-0.021}^{+0.010} \quad \mathrm{mag}, \quad c_{2}=1.36_{-0.20}^{+0.17}, \quad c_{3}<0.12, \quad c_{4}=$ $2.02_{-0.39}^{+0.34}$, and $\sigma=0.042_{-0.005}^{+0.005} \mathrm{mag}$. We plot the best-fit UBVRI light curves in Figure 3, and the best-fit spectral flux distribution, convolved with a canonical filter function, for two epochs in Figure 4. In Figure 4, we plot the effective spectral flux $1.21 \pm 0.04 \mu \mathrm{Jy}$ and frequency $1.09 \times 10^{15} \mathrm{~Hz}$ of the STIS measurement for our best-fit spectrum. However, the uncertainty in our fitted spectrum is not reflected in these values and uncertainties, and consequently, they should not be used in modeling efforts. Rather, the measured count rate should be fitted to using equation (2).

These results are consistent with the results of Lee et al. (2001) for their assumed spectral slope of $\beta=-0.75$ : Lee et al. (2001) find that $A_{V}<0.06$ mag at the $1 \sigma$ confidence level

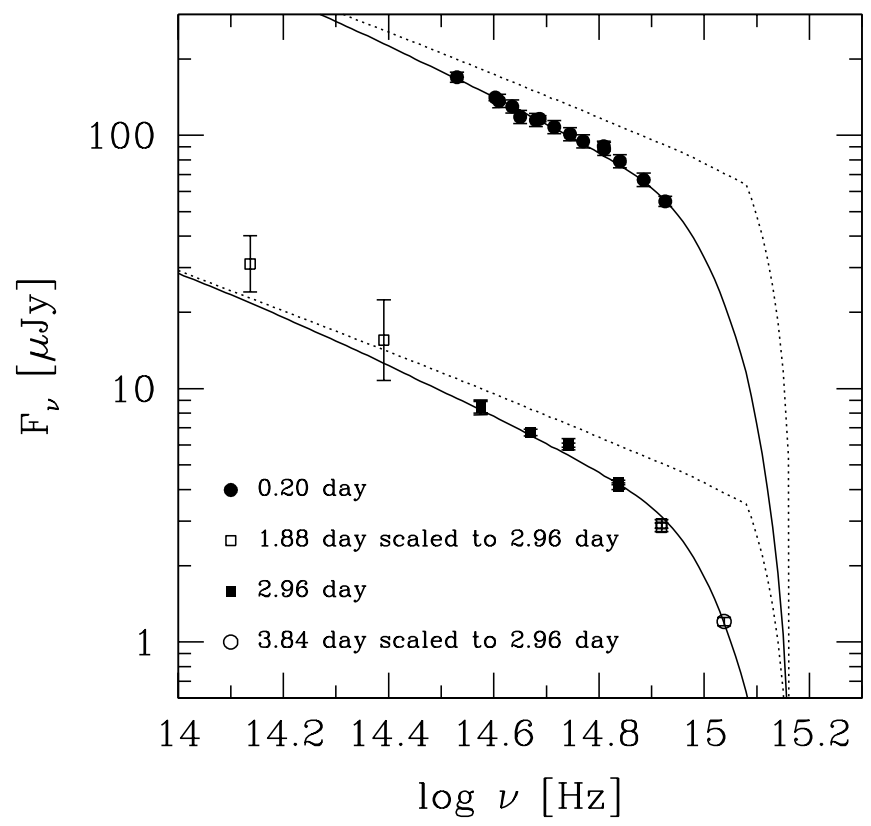

Fig. 4.-Spectral flux distribution at 0.20 and 2.95 days after the event. Data are from Lee et al. (2001) and Jha et al. (2001, filled circles), Masetti et al. (2001, filled and open squares), and this work (STIS NUV MAMA, open circle). Shown is the assumed spectral flux distribution, a power law with index $\beta$, that is modified by the Ly $\alpha$ break and forest and small Galactic foreground extinction (dotted curves). The index $\beta=p / 2$ is determined from a global fit to all optical data. The fit (solid curves) accounts for additional extinction in the rest frame of the OT and employs the full extinction curve model of Reichart (2001). See $\S 3.2$ for details. and less than $0.27 \mathrm{mag}$ at the $2 \sigma$ confidence level, but greater than $0 \mathrm{mag}$ at the $4.3 \sigma$ confidence level. We find that $A_{V}>0$ mag at the $5.4 \sigma$ confidence level. Furthermore, Lee et al. (2001) find that $c_{2}=1.35_{-0.21}^{+0.18}$ and $c_{4}>0$ at the $2.5 \sigma$ confidence level, and $c_{4}>1$ - the largest value previously observed - at the $2.1 \sigma$ confidence level. We find that $c_{4}>0$ at the $5.3 \sigma$ confidence level, and $c_{4}>1$ at the $2.8 \sigma$ confidence level. A canonical value for $c_{4}$ is about 0.5 , and $c_{4}$ ranges between 0 and 1 in the Milky Way, the LMC, and the SMC (Reichart 2001). Lee et al. (2001) suggest that this stronger-than-expected FUV excess component of the extinction curve might be due to sublimation and fragmentation of circumburst dust (e.g., Waxman \& Draine 2000; Galama \& Wijers 2001); if small (radius $<300 \AA$ ) graphite grains, which are probably responsible for the FUV excess component of the extinction curve (e.g., Draine \& Lee 1984), survive in greater numbers than other grains, this value of $c_{4}$ would not be unexpected. This result is seemingly at odds with the recent work of Perna, Lazzati, \& Fiore (2003), who have modeled the temporal evolution of the dust properties subject to a strong X-ray/UV radiation field. They find that the dust destruction preferentially occurs through sublimation of smaller grains, leading to a flat extinction curve.

Predehl \& Schmitt (1995) have measured the relation between optical extinction and hydrogen column for the Milky Way. Assuming a Galactic relation between $A_{V}$ and $N_{\mathrm{H}}$, our measurement of $A_{V}=0.110_{-0.021}^{+0.010}$ mag corresponds to a hydrogen column of $N_{\mathrm{H}}=1.8 \times 10^{20} \mathrm{~cm}^{-2}$. The hydrogen column density is found to be $N_{\mathrm{H}}=$ $(1.5 \pm 0.3) \times 10^{21} \mathrm{~cm}^{-2}$ (in 't Zand et al. 2001), corresponding to a rest-frame column density of $N_{\mathrm{H}}=(1.4 \pm 0.3) \times$ $10^{22} \mathrm{~cm}^{-2}$, i.e., $78 \pm 17$ times larger than expected on the basis of the Galactic relation. For the value of $N_{\mathrm{H}}$ derived from Chandra observations by Björnsson et al. (2002), this ratio is about a factor of 2 lower.

\section{DISCUSSION AND CONCLUSIONS}

The late-time optical observations with WFPC2 on HST flatten after $\sim 25$ days, indicating the presence of a host galaxy; we derive from the WFPC2 images $B_{\text {gal }}=$ $25.71_{-0.24}^{+0.32}, \quad V_{\mathrm{gal}}=26.18_{-0.13}^{+0.15}, R_{\mathrm{gal}}=26.42_{-0.21}^{+0.27}$, and $I_{\mathrm{gal}}=$ $25.66_{-0.24}^{+0.31}$ mag. We find that the host galaxy emission is dominated by a very compact core, similar to, for example, the host of GRB 970508 (Fruchter et al. 2000). Similar conclusions about the host galaxy properties were also reached by Fruchter et al. (2001b) from an independent analysis of these same data.

These late-time optical observations also show that the optical light curves continued their decay slightly faster; we find a significantly larger value of the electron energy index $p=1.57_{-0.03}^{+0.04}$ and a later jet-break time $t_{*}=0.93_{-0.06}^{+0.15}$ days than previous work (Cowsik et al. 2001; Masetti et al. 2001; Sagar et al. 2001; Stanek et al. 2001) has reported ( $p \sim 1.4$ and $t_{*} \sim 0.6$ days). At the same time, the quality of the fit is good (requiring a modest value for the slop parameter, $\sigma$ ) and indicates that a single value of $p$ suffices to describe the observations. We are not required by the observations to resort to a more complicated model with, e.g., an index, $p$, that increases with time (reflected in an increase in decay rate with time). Our finding of a steeper decay rate may be caused by (1) our detection of and fitting for a host 
galaxy contributing to the late-time emission, which suggests that previous work requires a small correction for its contribution; and (2) the jet transition may not yet have been fully developed in the early-time observations presented by previous workers (resulting in their finding a lower $p$ ). For accurate determination of the physical parameters of the afterglow, late-time sensitive observations with $H S T$ are of great importance. Our determination of $p$ therefore supersedes that of previous work.

Our STIS NUV measurement indicates that the spectral flux distribution falls off rapidly toward the ultraviolet; the far-ultraviolet (FUV) curvature component $c_{4}$ (Reichart 2001 ) is rather large. This may be due to dust destruction, where larger grains are preferentially destroyed. This provides further support for the idea that the early, hard radiation from the GRB and its afterglow should destroy dust in the circumburst environment, carving a path out of the molecular cloud through which later afterglow light can travel relatively unobstructed (Waxman \& Draine 2000; Fruchter et al. 2001a).

Additional observational support for dust destruction comes from the fact that the hydrogen column density is found to be $\sim 40-80$ times larger than expected from the observed $A_{V}$. This appears to be a trend in GRB afterglows; the column densities are high and found to be typical of Galactic giant molecular clouds (Galama \& Wijers 2001; Reichart \& Price 2002), but the optical extinction is found to be small (factors of 10-100 times smaller). Galama \& Wijers (2001) have interpreted this as evidence that GRBs occur in dense star-forming regions and that the dust is indeed being destroyed.

Also, from the possible connection of GRBs with supernovae (Galama et al. 1998, 2000; Kulkarni et al. 1998; Bloom et al. 1999, 2002b; Reichart 1999; Castro-Tirado et al. 2001; Björnsson et al. 2001), we would expect that GRBs occur in dense star-forming regions; large amounts of optical extinction are then naturally expected, but only small amounts are observed (Galama \& Wijers 2001).

On the other hand, early-time Keck spectroscopic observations of GRB 010222 (Mirabal et al. 2002) show a two-component system similar to that of GRB 000926 (Castro et al. 2002). In GR B 000926 the two absorption systems have a velocity separation of $168 \mathrm{~km} \mathrm{~s}^{-1}$, which is interpreted as being due to two individual clouds in the host galaxy. Dust destruction by the GRB and the afterglow is effective only to $\sim 10-100$ pc (Waxman \& Draine 2000; Fruchter et al. 2001a) from the GRB, and it is therefore unlikely that the dust in both clouds would be destroyed. Further, the two clouds appear to have similar relative metal abundance and dust-to-gas ratio. Castro et al. (2002) argue that one of the clouds is probably associated with the GRB site and, consequently, that the burst was ineffective at sublimating dust, contradicting theoretical expectations (e.g., Waxman \& Draine 2000). In other words, the explanation for the observed low optical extinctions could also be that GRB host galaxies typically have low dust-to-gas ratios (cf. Pettini et al. 1997). This is a simple and attractive explanation, based only on the assumption that the dust-togas ratio was the same for both clouds before the burst occurred. However, in higher redshift galaxies, such as this one, the dust-to-gas ratio might be high only in those clouds that are actively producing stars, which is presumably the case at the GRB site (i.e., dust has not had sufficient time to mix with gas elsewhere in the galaxy). The burst would sublimate this dust, returning the metals to the gas phase, and consequently, similar relative metallicities and dust-to-gas ratios might also be interpreted as evidence in favor of sublimation.

E. O. Ofek and J. Dann are thanked for their help with observations at the Wise Observatory. We wish to thank Steve Beckwith and the HST operations staff for facilitating the WFPC2 and STIS observations. T. J. G. acknowledges support from the Sherman Fairchild Foundation. F. A. H. acknowledges support from a Presidential Early Career award. S. R. K. and S. G. D. thank the National Science Foundation for support of their ground-based GRB programs. A. G. J. is a Colton Fellow. J. S. B. is a Fannie and John Hertz Foundation Fellow.
Berger, E., \& Frail, D. 2001, GCN Circ. 968 (http://gcn.gsfc.nasa.gov/ $\mathrm{gcn} / \mathrm{gcn} 3 / 968 . \mathrm{gcn} 3)$

Bessell, M. S. 1979, PASP, 91, 589

Bessell, M. S. \& Brett, J. M. 1988, PASP, 100, 1134

Bhattacharya, D. 2001, Bull. Astron. Soc. India, 29, 107

Björnsson, G., Hjorth, J., Jakobsson, P., Christensen, L., \& Holland, S. 2001, ApJ, 552, L121

Björnsson, G., Hjorth, J., Pedersen, K., \& Fynbo, J. U. 2002, ApJ, 579, L59

Bloom, J. S. 2003, PASP, in press

Bloom, J. S., Kulkarni, S. R., \& Djorgovski, S. G. 2002a, AJ, 123, 1111

Bloom, J. S., et al. 1999, Nature, 401, 453

2001, GCN Circ. 989 (http://gcn.gsfc.nasa.gov/gcn/gcn3/ 989. $g$ cn 3$)$ 2002b, ApJ, 572, L45

Cardelli, J. A., Clayton, G. C., \& Mathis, J. S. 1989, ApJ, 345, 245

Castro, S., et al. 2001, GCN Circ. 999 (http://gcn.gsfc.nasa.gov/gcn/ $\operatorname{gcn} 3 / 999 . \operatorname{gcn} 3)$

2002, ApJ, submitted (astro-ph/0110566)

Castro-Tirado, A. J., et al. 2001, A\&A, 370, 398

Cowsik, R., Prabhu, T. P., Anupama, G. C., Bhatt, B. C., Sahu, D. K., Ambika, S., Padmakar, \& Bhargavi, S. G. 2001, Bull. Astron. Soc. India, 29,157

Dai, Z. G., \& Cheng, K. S. 2001, ApJ, 558, L109

Draine, B. T., \& Lee, H. M. 1984, ApJ, 285, 89

Fich, M., et al. 2001, GCN Circ. 971 (http://gcn.gsfc.nasa.gov/gen/gen3/ 971.gen 3 )

Frail, D. A., et al. 2002, ApJ, 565, 829

Fruchter, A. S., \& Hook, R. N. 2002, PASP, 114, 144

Fruchter, A. S., Krolik, J. H., \& Rhoads, J. E. 2001a, ApJ, 563, 597

Fruchter, A. S., et al. 2000, ApJ, 545, 664

\section{RFERENCES}

Fruchter, A. S., et al. 2001b, GCN Circ. 1087 (http://gcn.gsfc.nasa.gov/ $\mathrm{gcn} / \mathrm{gcn} 3 / 1087 . \mathrm{gcn} 3)$

Fukugita, M., Ichikawa, T., Gunn, J. E., Doi, M., Shimasaku, K., \& Schneider, D. P. 1996, AJ, 111, 1748

Galama, T. J., \& Wijers, R. A. M. J. 2001, ApJ, 549, L209

Galama, T. J., et al. 1998, Nature, 395, 670

2000, ApJ, 536, 185

Garnavich, P. M., et al. 2001, GCN Circ. 965 (http://gcn.gsfc.nasa.gov/ $\mathrm{gcn} / \mathrm{gcn} 3 / 965 . \mathrm{gcn} 3)$

Harrison, F. A., et al. 2001, ApJ, 559, 123

Henden, A. 2001a, GCN Circ. 962 (http://gcn.gsfc.nasa.gov/gcn/gcn3/ 962.gcn 3 )

. 2001b, GCN Circ. 987 (http://gcn.gsfc.nasa.gov/gcn/gcn3/ 987. $\operatorname{gcn} 3)$

Henden, A., \& Vrba, F. 2001, GCN Circ. 967 (http://gcn.gsfc.nasa.gov/ $\mathrm{gcn} / \mathrm{gcn} 3 / 967 . \mathrm{gcn} 3$ )

Holland, S., et al. 2001, GCN Circ. 1002 (http://gcn.gsfc.nasa.gov/gcn/ gen $3 / 1002 . g c n 3)$

Holtzman, J. A., Burrows, C. J., Casertano, S., Hester, J. J., Trauger, J. T., Watson, A. M., \& Worthey, G. 1995, PASP, 107, 1065

in 't Zand, J. J. M., et al. 2001, ApJ, 559, 710

Jha, S., et al. 2001, ApJ, 554, L155

Kulkarni, S. R., et al. 1998, Nature, 395, 663

Lee, B. C., et al. 2001, ApJ, 561, 183

Masetti, N., et al. 2001, A\&A, 374, 382

Massi, F., Dolci, M., \& Carlo, E. D. 2001, GCN Circ. 980 (http:// gcn.gsfc.nasa.gov/gcn $/ \mathrm{gcn} 3 / 980 . \mathrm{gcn} 3$ )

McDowell, J., et al. 2001, GCN Circ. 963 (http://gcn.gsfc.nasa.gov/gcn/ gcn3/963.gcn3)

Mirabal, N., et al. 2002, ApJ, 578, 818 
Oksanen, A., et al. 2001, GCN Circ. 990 (http://gcn.gsfc.nasa.gov/gcn/ $\operatorname{gcn} 3 / 990 . \operatorname{gcn} 3)$

Orosz, J. 2001, GCN Circ. 976 (http://gcn.gsfc.nasa.gov/gcn/gcn3/ 976.gen 3 )

Panaitescu, A. 2001, ApJ, 556, 1002

Panaitescu, A., \& Kumar, P. 2002, ApJ, 571, 779

Perna, R., Lazzati, D. \& Fiore, F. 2003, ApJ, 585, 774

Pettini, M., Smith, L. J., King, D. L., \& Hunstead, R. W. 1997, ApJ, 486, 665

Piro, L. 2001a, GCN Circ. 960 (http://gcn.gsfc.nasa.gov/gcn/gcn3/ 960. $\operatorname{gcn} 3$ ) 966.gcn3)

Piro, L., et al. 2001, ApJ, 558, 442

Predehl, P., \& Schmitt, J. H. M. M. 1995, A\&A, 293, 889

Reichart, D. E. 1999, ApJ, 521, L111
Reichart, D. E. 2001, ApJ, 553, 235

Reichart, D. E., \& Price, P. A. 2002, ApJ, 565, 174

Rhoads, J. E. 1999, ApJ, 525, 737

Sagar, R., et al. 2001, Bull. Astron. Soc. India, 29, 91

Salamanca, I., et al. 2002, MNRAS, submitted (astro-ph/0112066)

Sari, R., Piran, T., \& Halpern, J. P. 1999, ApJ, 519, L17

Sari, R., Piran, T., \& Narayan, R. 1998, ApJ, 497, L17

Schlegel, D. J., Finkbeiner, D. P., \& Davis, M. 1998, ApJ, 500, 525

Stanek, K. Z., et al. 2001, ApJ, 563, 592

Veillet, C. 2001a, GCN Circ. 1000 (http://gcn.gsfc.nasa.gov/gcn/gcn3/ $1000 . \mathrm{gcn} 3)$ 2001b, GCN Circ. 1003 (http://gcn.gsfc.nasa.gov/gcn/gcn3/ 1003.gcn3)

Watanabe, J., Kinoshita, D., Komiyama, Y., Fuse, T., Urata, Y., \& Yoshida, F. 2001, PASJ, 53, L27

Waxman, E., \& Draine, B. T. 2000, ApJ, 537, 796 